\title{
Increased expression of p38 MAPK in human bronchial epithelium after
} lipopolysaccharide exposure

\author{
E. Roos-Engstrand*, A. Wallin*, A. Bucht*\#, J. Pourazar*, \\ T. Sandström* and A. Blomberg*
}

ABSTRACT: Bacterial endotoxin (lipopolysaccharides (LPS)) is normally present in the wall of Gram-negative bacteria and has potent pro-inflammatory properties. Exposure to LPS has been shown to induce neutrophilic airway inflammation in humans. The aim of this investigation was to study the early inflammatory responses to LPS exposure in human airway mucosa in vivo.

In total, 15 healthy nonsmoking volunteers participated. Bronchoscopy was performed on two separate occasions, $3 \mathrm{~h}$ after saline inhalation and after inhalation of $50 \mu \mathrm{g}$ LPS in saline. Endobronchial mucosal biopsy specimens were taken and stained immunohistochemically using a panel of monoclonal antibodies directed against mitogen-activated protein kinases (MAPKs), transcription factors, cytokines, adhesion molecules and inflammatory cells.

Expression of p38 MAPK increased as a consequence of LPS exposure, as determined by both total epithelial staining and nuclear location. These two responses were strongly associated. Epithelial expression of interleukin-8 showed a tendency towards a significant increase after LPS compared to saline. Epithelial mast cell numbers were increased after LPS, whereas neutrophil numbers were unchanged.

Inhalation of lipopolysaccharide induced activation of the bronchial epithelium, as demonstrated $3 \mathrm{~h}$ after exposure by increased expression of p38 mitogen-activated protein kinase and interleukin-8, and may represent early regulatory steps in the subsequent development of a neutrophilic bronchial inflammation.

KEYWORDS: Airway inflammation, interleukin-8, lipopolysaccharide, p38 mitogen-activated protein kinase

ndotoxin and its purified derivative lipopolysaccharides (LPS) are glycolipids normally present in the cell wall of Gramnegative bacteria and are potent pro-inflammatory substances. LPS are continuously shed into the surrounding environment and are present in organic dust and found in places such as poultry houses, cotton mills, swine confinement buildings, sawmills and many other locations. Inhalation of organic dust has been shown to induce systemic and airway inflammation, and several studies have been carried out in healthy subjects to address the systemic and local response to LPS [1, 2]. These studies have reported headache, fever, cough, chest tightness

For editorial comments see page 776 . and increased bronchial reactivity, as well as reduced forced expiratory volume in one second (FEV1), as a consequence of LPS exposure [3, 4].

It has previously been reported that LPS exposure is capable of inducing pronounced airway inflammation in terms of increased numbers of polymorphonuclear neutrophils and lymphocytes, as well as increased fibronectin concentration in bronchoalveolar lavage (BAL) fluid from healthy subjects, as early as $3 \mathrm{~h}$ after LPS challenge [5]. MicHel et al. [6] have reported increased numbers of total white blood cells and polymorphonuclear neutrophils in peripheral blood after LPS exposure of normal subjects. In induced sputum, neutrophils, monocytes, lymphocytes, myeloperoxidase, eosinophilic cationic protein, tumour necrosis factor- $\alpha$ (TNF- $\alpha$ ) and
AFFILIATIONS

*Dept of Respiratory Medicine and Allergy, University Hospital, and \#Dept of Medical Countermeasures, Swedish Defence Research Agency, NBC Defence, Umeå, Sweden.

CORRESPONDENCE

A. Blomberg

Dept of Respiratory Medicine and Allergy

University Hospital

SE-901 85 Umeå

Sweden

Fax: 4690141369

E-mail:

anders.blomberg@lung.umu.se

Received

June 302004

Accepted after revision:

December 082004

SUPPORT STATEMENT

This study was supported by GlaxoSmithKline (MöIndal, Sweden) and the Swedish Heart Lung

Foundation (Stockholm, Sweden).
European Respiratory Journal Print ISSN 0903-1936 Online ISSN 1399-3003 
interleukin (IL)-8 levels have been found to increase after inhalation of LPS [7].

LPS bind to LPS-binding protein and are delivered to the cell surface receptor CD14. LPS are then transferred to the transmembrane signalling receptor Toll-like receptor (TLR)-4. Several in vitro and animal studies have addressed the early inflammatory responses to LPS. In LPS-stimulated alveolar macrophages, CARTER et al. [8] found that both extracellular signal-regulated protein kinase (ERK) and p38 mitogenactivated protein kinase (MAPK) pathways play a critical role in the inflammatory response in sepsis-induced acute lung injury. Stimulation with LPS of human monocytes in vitro has been shown to activate several intracellular signalling pathways, such as nuclear factor- $\kappa \mathrm{B}(\mathrm{NF}-\kappa \mathrm{B})$ and MAPK pathways, such as ERK 1 and 2, c-jun N-terminal kinase (JNK) and p38 [9]. Furthermore, LPS have been shown to stimulate p38 MAPK and IL-8 production in human mononuclear cells [10].

To date, there are no studies addressing the early inflammatory responses to LPS in human airway tissue in vivo in healthy volunteers. The aim of the present investigation was, therefore, to study the early LPS-induced airway inflammatory responses in vivo with specific reference to epithelial cell expression of MAPKs, transcription factors, cytokines and inflammatory cells. It was hypothesised that LPS exposure would induce upregulation of MAPKs, transcription factors and cytokines in the bronchial epithelium, as an early inflammatory response leading to the subsequent neutrophil recruitment.

\section{MATERIAL AND METHODS \\ Subjects}

In total, 15 healthy nonsmoking volunteers (eight males and seven females; mean age 25 yrs (range 21-38 yrs)) without any history of allergy, asthma or other pulmonary diseases participated. No respiratory infection was permitted within $\geqslant 4$ weeks before or during the study. The study was approved by the local ethics committee of the University of Umeå (Umeå, Sweden), and written informed consent was given by each volunteer.

\section{Study design: lipopolysaccharide exposure}

LPS inhalation was performed using LPS from Escherichia coli (serotype 026:B6) prepared using a phenol extraction procedure (Sigma Chemical Co., St Louis, MO, USA). The subjects inhaled $50 \mu \mathrm{g}$ LPS suspended in $2 \mathrm{~mL}$ saline over a period of 10-15 min using a Pari-Boy inhaler (Pari, Starnberg, Germany). The Pari-Boy nebuliser has been shown to produce a particle size with a mass median diameter of $3.5 \mu \mathrm{m}$ (range $0.5-$ $5.5 \mu \mathrm{m}$ ). As the dose of LPS reaching the lung is $\sim 50 \%$ of the total dose [3], the pulmonary dose in this case would be $25 \mu \mathrm{g}$, clearly sufficient to induce pronounced neutrophilic airway inflammation, as shown in BAL fluid [11]. Inhalation of saline was performed $\geqslant 3$ weeks apart from the LPS inhalation and used as a reference. Thus, each volunteer acted as their own control.

\section{Methods}

\section{Bronchoscopy}

Pre-medication with atropine $(0.5-1.0 \mathrm{mg})$ was given subcutaneously, and lidocaine was used for topical anaesthesia of the airways as previously reported [12]. Three hours after each inhalation, the subjects were examined in the supine position using an Olympus BF T10 or BF T20 fibreoptic bronchoscope (Olympus, Tokyo, Japan). During each bronchoscopy procedure, three endobronchial mucosal biopsy specimens were obtained according to a standard protocol [12]. The timing of the bronchoscopy was based on a previous investigation in which pronounced neutrophilic inflammation was reported in BAL fluid $3 \mathrm{~h}$ after LPS inhalation [5]. Endobronchial mucosal biopsy sampling was randomised to either the subcarinae of the third- and fourth-generation bronchi of the right or left side during the first bronchoscopy procedure. In order to avoid artefacts from the former biopsy sites, the contralateral lung was used for biopsy sampling during the second bronchoscopy procedure. As previously reported, a bronchial wash (BW) with $2 \times 20 \mathrm{~mL}$ and BAL with $3 \times 60 \mathrm{~mL}$ sterile phosphatebuffered saline at $37^{\circ} \mathrm{C}$ were also performed [11]. Lavages were carried out on the opposite lung, i.e. a lingular lobe bronchus when biopsy specimens were taken from the right side and a middle-lobe bronchus when biopsy specimens were taken from the left side.

\section{Processing and immunostaining of glycol methacrylate- embedded tissue sections}

The tissue biopsy specimens were placed in ice-cooled acetone containing the protease inhibitors phenylmethylsulphonyl fluoride $(2 \mathrm{mM})$ and iodoacetamide $(20 \mathrm{mM})$. The biopsy specimens were further processed into glycol methacrylate resin as previously described [13]. The glycol methacrylate blocks were then stored in airtight containers at $-20^{\circ} \mathrm{C}$ until they were used for immunostaining. First, sections from each block were stained with toluidine blue, in order to estimate the quality of the submucosa and epithelium of the biopsy specimens. Sections from the glycol methacrylate block with the best morphology were cut and stained with monoclonal antibodies (mAbs) as previously outlined [12]. In short, 2- $\mu \mathrm{m}$ thick sections were cut and treated to block endogenous peroxidase activity. Nonspecific antibody binding was blocked using a solution containing $0.1 \%$ sodium azide and $0.3 \%$ hydrogen peroxide, and the sections washed three times in tris(hydroxymethyl)aminomethane (Tris)-buffered saline (TBS) adjusted to $\mathrm{pH}$ 7.6. Undiluted blocking medium, consisting of Dulbecco's minimal essential medium (DMEM) containing $10 \%$ foetal calf serum and $1 \%$ bovine serum albumin (BSA), was applied for $30 \mathrm{~min}$ and then drained off. Mouse mAbs (table 1) were added to the sections and incubated overnight (16-20 h) at room temperature. After three washes with TBS, biotinylated rabbit anti-mouse immunoglobulin $\mathrm{G} \quad \mathrm{F}\left(\mathrm{ab}^{\prime}\right)_{2}$ (Dako, Glostrup, Denmark) was applied to the sections for $2 \mathrm{~h}$. This was followed by three washes in TBS and then streptavidin-biotin-horseradish peroxidase complex (Dako) was added for a further $2 \mathrm{~h}$. Finally, after three washes in TBS, positive immunoreactions for cell markers and adhesion molecules were developed using aminoethyl carbazole in acetate buffer ( $\mathrm{pH}$ 5.2) and hydrogen peroxide to yield a red colour. Sections analysed for cytokine expression in the bronchial epithelium were developed using 3,3'-diaminobenzidine tetrahydrochloride (DAB) as a substrate to yield a brown colour, as described previously [14]. All sections were counterstained with Mayer's haematoxylin. Sections where the primary antibody was omitted served as negative controls. 
TABLE 1 Monoclonal antibodies\# used in the immunohistochemical analysis

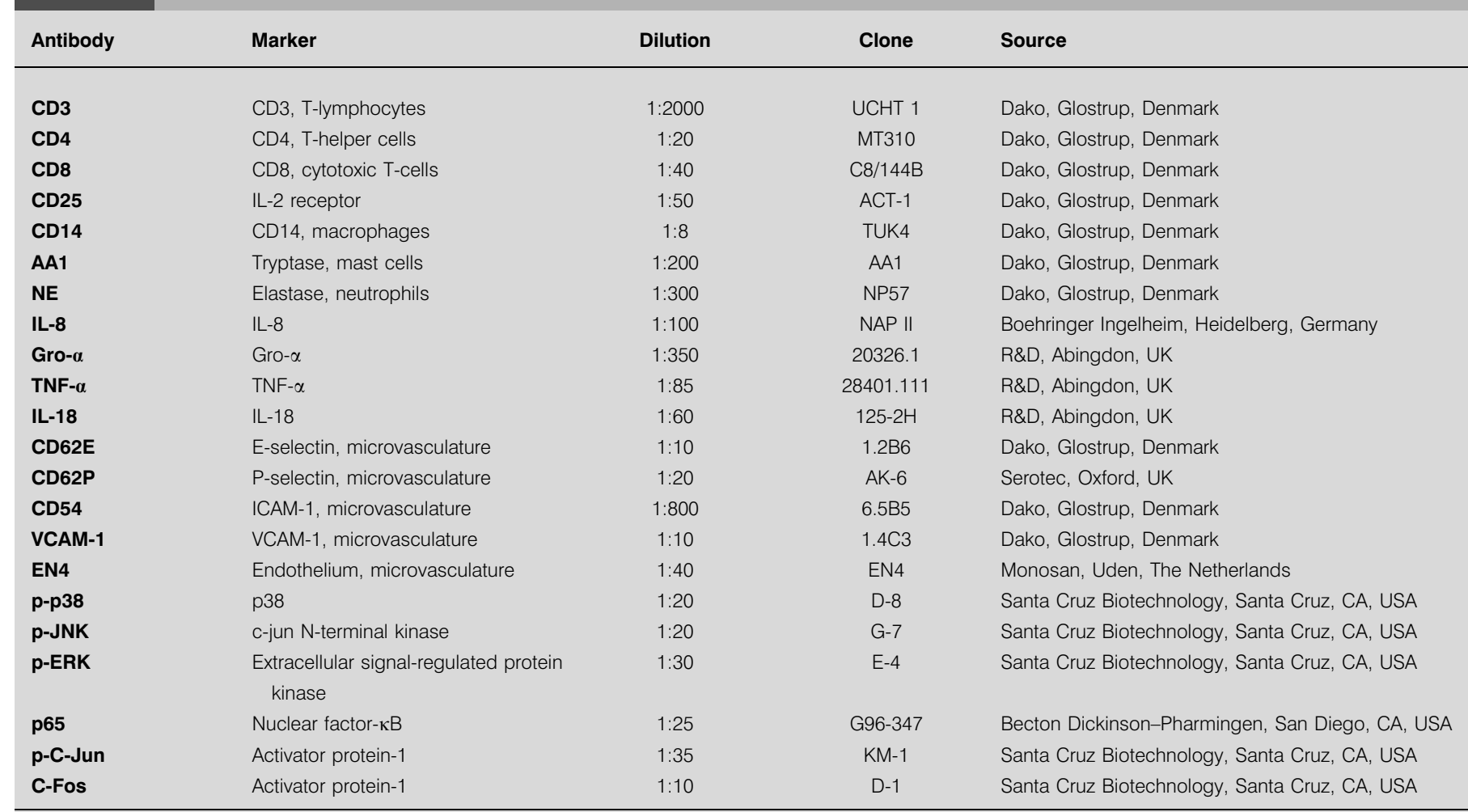

IL: interleukin; Gro: growth-related oncogene; TNF: tumour necrosis factor; ICAM: intercellular adhesion molecule; VCAM: vascular cell adhesion molecule. \#: against inflammatory cells, cytokines, adhesion molecules, mitogen-activated protein kinases and transcription factors.

The staining procedure was partially modified for transcription factors and MAPKs. Triton X (VWR International, Darmstadt, Germany) $0.3 \%$ in TBS was applied to the sections for $30 \mathrm{~min}$ in order to increase the permeability of the cells. After the first blocking step with DMEM, an additional blocking step was performed using rabbit normal serum for $30 \mathrm{~min}$. mAbs were diluted in $0.05 \%$ Triton $X$ in TBS containing $1 \%$ BSA. The overnight incubation was followed by further washes with $0.1 \%$ Triton $X$ in TBS. Streptavidin-biotin-horseradish peroxidase complex was added for a further $2 \mathrm{~h}$. After washing in $0.1 \%$ Triton $\mathrm{X}$ in TBS, a positive immunoreaction was visualised as a brown colour with DAB and the sections were counterstained with Mayer's haematoxylin. The better of two stained sections from each bronchoscopic procedure was analysed.

\section{Quantification of immunostaining in mucosal biopsy specimens}

Cells

Quantification was performed using a light microscope. For each $\mathrm{mAb}$, the number of inflammatory cells staining positively was determined separately in the epithelium and in the submucosa, excluding mucosal glands, large blood vessels and smooth muscle. The epithelium was considered to be the area of epithelial cells above the basement membrane. The total number of positive cells was expressed as cells per millimetre and cells per millilitre squared in the epithelium and submucosa, respectively. The length of the epithelium and the area of the submucosa were calculated using computer-assisted image analysis (Qwin, Leica Q500IW; Leica, Cambridge, UK). The cells showed two different staining patterns, i.e. cytoplasmic staining (neutrophil elastase and mast cell tryptase) and ring staining (CD3, CD4, CD8, CD14 and CD25).

\section{Adhesion molecules}

Quantification of endothelial adhesion molecules in the submucosal blood vessels was carried out by expressing the number of vessels staining with specific anti-adhesion molecule $\mathrm{mAbs}$ as a percentage of the total vessel complement, revealed by staining with the panendothelial mAb EN4 [15].

\section{Cytokines}

Quantification of cytokine immunoreactivity in the bronchial epithelium was performed on sections developed with DAB [16]. The epithelial expression of each cytokine was quantified with the assistance of computer-assisted image analysis and expressed as the percentage of the total epithelial area showing positive immunostaining. Only areas of intact epithelium, with cilia and basal membrane present, were included in the analysis.

\section{Mitogen-activated protein kinases and transcription factors}

Quantification of phosphorylated MAPK and transcription factor immunoreactivity in the bronchial epithelium was performed on sections developed with DAB [16]. Total staining (cytoplasmic and nuclear) was expressed as the percentage of the total epithelial area showing positive immunostaining, using image analysis. 
As it was clearly possible to distinguish between nuclear and cytoplasmic staining, quantification of nuclear staining was performed using a light microscope. Positive staining of the nucleus or nuclear membrane was expressed in terms of the number of positive nuclei per millimetre squared of epithelium.

The median epithelial area analysed for cytokines, transcription factors and MAPKs was $102,000 \mu \mathrm{m}^{2}$ (interquartile range $\left.67,865-120,222 \mu \mathrm{m}^{2}\right)$. For cells, the submucosal area analysed was $0.506(0.332-0.724) \mathrm{mm}^{2}$ and the epithelial length 1,786 $(1,492-2,470) \mu \mathrm{m}$.

Analyses of bronchial wash and bronchoalveolar lavage fluids IL-6, IL- 8 and TNF- $\alpha$ were measured using commercially available ELISA kits (R\&D Systems, Abingdon, UK).

\section{Statistical analysis}

The subjects acted as their own controls. The Wilcoxon nonparametric signed-rank test for paired observations was employed. A p-value of $<0.05$ was considered significant. Correlation analysis was carried out using Spearman's rankorder correlation and a p-value of $<0.01$ was considered significant.

\section{RESULTS}

As reported in a previous publication, the present LPS dose induced no symptoms and no effects on FEV1, but a significant increase in airway neutrophil numbers, as reflected in both BW and BAL fluid [11].

Expression of phosphorylated p38 MAPK increased as a consequence of LPS exposure, both when determined as total epithelial staining (median (interquartile range) 0.63 (0.35-1.57) versus $0.23(0.11-0.33) \% ; p=0.006)$ and as nuclear staining (446 (246-780) versus $158(100-271)$ cells $\left.\cdot \mathrm{mm}^{-2} ; \mathrm{p}=0.009\right)$ after LPS versus saline exposure (table 2 , fig. 1 ). These two responses were strongly associated ( $\mathrm{r} s=0.864 ; \mathrm{p}<0.01$; fig. 2 ). No significant change in epithelial expression was noticed for JNK or ERK. In contrast, LPS exposure induced a significant decrease in nuclear c-Fos expression compared to control exposure (66 (12-99) versus $92(32-212)$ cells $\left.\cdot \mathrm{mm}^{-2} ; \mathrm{p}=0.03\right)$. Expression of $\mathrm{NF}-\kappa \mathrm{B}$ and $\mathrm{c}-\mathrm{Jun}$ was not significantly altered.

Epithelial expression of IL-8 showed a tendency towards a significant increase after LPS exposure compared to control (0.07 (0.00-0.56) versus $0.01(0.00-0.03) \% ; p=0.05)$. Expression of no other cytokines, including TNF- $\alpha$, was significantly changed. Nor were any significant changes found in the expression of vascular endothelial adhesion molecules. Epithelial mast cell numbers were significantly increased after exposure to LPS compared to saline $(0.55$ (0.00-1.43) versus 0.0 $(0.00-0.052)$ cells $\left.\cdot \mathrm{mm}^{-1} ; \mathrm{p}=0.03\right)$. The numbers of neutrophils and other inflammatory cells remained unchanged in both the epithelium and the submucosa (data not shown).

LPS challenge induced pronounced increases in BW fluid concentrations of TNF- $\alpha \quad(2.4 \quad(1.3-5.1)$ versus 0.0 $\left.(0.0-0.0) \mathrm{pg} \cdot \mathrm{mL}^{-1} ; \mathrm{p}=0.001\right)$ and IL-6 (13.9 (13.1-14.0) versus $\left.5.2(2.8-9.8) \mathrm{pg} \cdot \mathrm{mL}^{-1} ; \mathrm{p}=0.001\right)$. Similar responses were detected in BAL fluid TNF- $\alpha(2.8(1.5-3.5)$ versus $0.0(0.0$ $\left.0.0) \mathrm{pg} \cdot \mathrm{mL}^{-1} ; \mathrm{p}=0.001\right)$ and IL-6 (14.0 (10.2-14.9) versus 1.7 (1.22.1) $\left.\mathrm{pg} \cdot \mathrm{mL}^{-1} ; \mathrm{p}=0.001\right)$. No significant changes were found in IL-8 in either BW or BAL fluid (data not shown).

No sex-based differences were found in either of the above mentioned markers (data not shown).

\section{DISCUSSION}

The present study is the first investigation addressing the early inflammatory responses in human bronchial mucosa after inhalation of LPS in vivo. It was demonstrated that inhalation of LPS significantly increased the total epithelial expression, as well as nuclear location, of phosphorylated p38 MAPK; this was accompanied by a small increase in the expression of the neutrophil chemotactic chemokine IL-8 in airway epithelium $3 \mathrm{~h}$ after exposure. These responses are suggested to represent early regulatory steps in the subsequent development of LPSinduced neutrophilic inflammation.

The MAPK pathways form a group of three main pathways, including ERK 1 and 2, and two stress-activated protein kinases designated JNK and p38 MAPK. It is suggested that p38 MAPK is involved in regulating cytokine expression by phosphorylation and nuclear translocation, and plays a central role in a wide range of inflammatory responses in many different cells, such as neutrophils and other leukocytes [17].

To date, little is known about the role of p38 MAPK in LPSinduced IL-8 expression in the human airway epithelium in vivo. In human endothelial cells, LPS stimulation in vitro has been reported to induce p38 MAPK phosphorylation and activity, and certain inhibitors have been found to inhibit LPSinduced IL-8 expression [18, 19]. Thus, taken together, these data point indirectly to the importance of p38 MAPK in the development of subsequent neutrophilic inflammation.

\begin{tabular}{|c|c|c|c|c|c|c|}
\hline & \multicolumn{2}{|c|}{ p38 } & \multicolumn{2}{|c|}{ JNK } & \multicolumn{2}{|c|}{ ERK } \\
\hline Placebo & $0.23(0.11-0.33)$ & $158(100-271)$ & $0.60(0.45-0.71)$ & $534(477-693)$ & $0.24(0.08-0.35)$ & 198 (90.3-386) \\
\hline LPS & $0.63(0.35-1.57)$ & 446 (246-780) & $0.75(0.27-0.82)$ & 475 (270-678) & $0.28(0.03-0.60)$ & 231 (75.8-374) \\
\hline
\end{tabular}

Data are presented as median (interquartile range), unless otherwise stated. JNK: c-jun N-terminal kinase; ERK: extracellular signal-regulated protein kinase; LPS: lipopolysaccharides. 

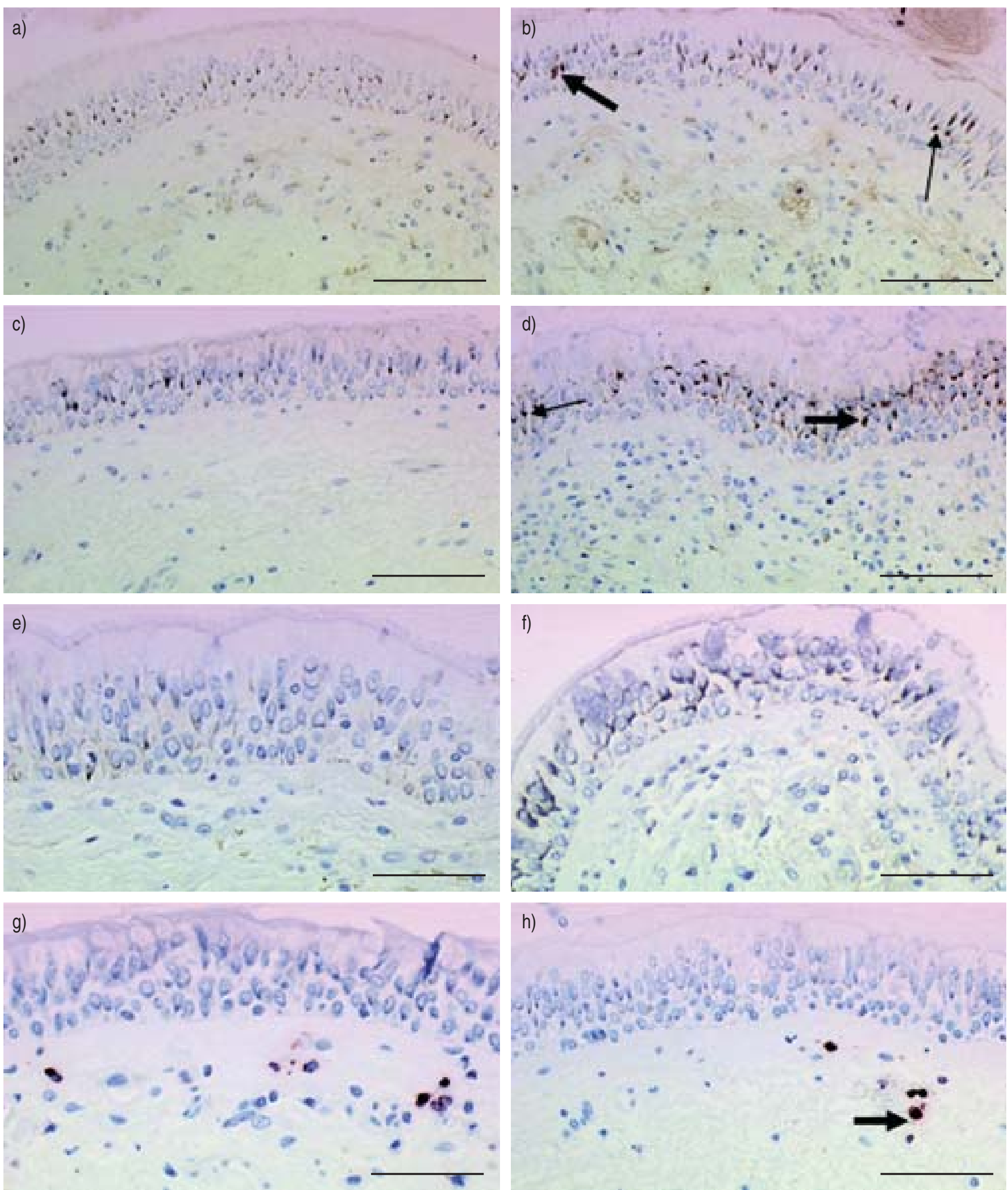

FIGURE 1. Immunohistochemical staining of bronchial tissue for: $a, b)$ p38 mitogen-activated protein kinase, $c$, d) nuclear factor-kB, e, f) interleukin-8, and $g$, h) neutrophils; after: a, c, e, g) saline inhalation, and b, d, f, h) lipopolysaccharides (LPS) inhalation. a-f) Brown areas represent positive staining; the thick arrow indicates nuclear staining and the thin arrow cytoplasmic staining. $\mathrm{g}$, h) Red granular staining indicated by thick arrow represents positive staining. Scale bars $=50 \mu \mathrm{m}$. 


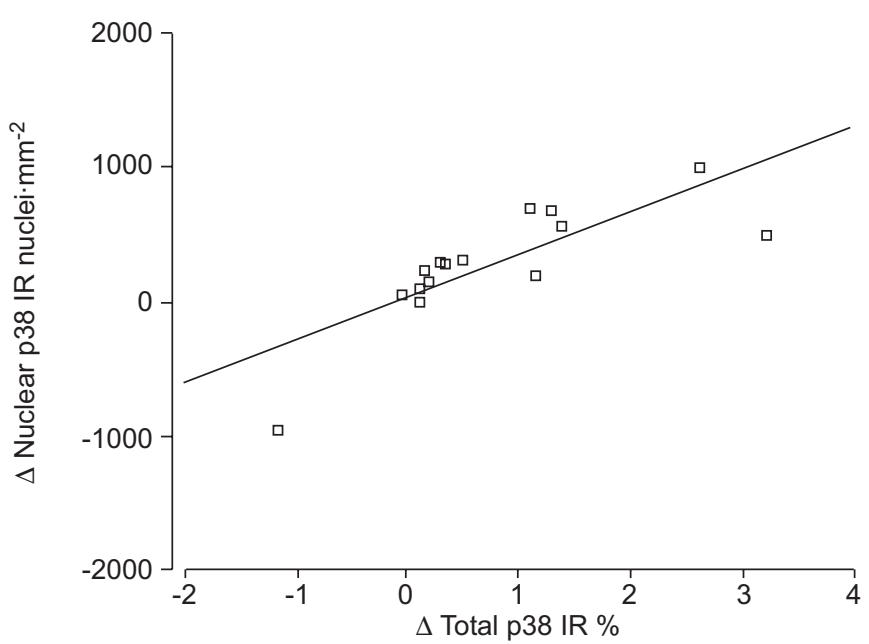

FIGURE 2. Correlation between lipopolysaccharides (LPS)-induced response (compared to saline) of total and nuclear p38 mitogen-activated protein kinase immunoreactivity (IR). $\Delta$ : change. $r_{s}=0.864 ; p<0.01$.

LPS inhalation increased phosphorylated p38 MAPK expression in both the nuclear and cytoplasmic compartments of the cells, suggesting LPS-induced synthesis of p38 MAPK as well as nuclear translocation of the protein. The positive association between the total epithelial expression and nuclear location of phosphorylated p38 MAPK indicates that the increased expression of nuclear p38 MAPK is a consequence of translocation of cytoplasmic p38 MAPK and, therefore, a sign of activation (fig. 2). Taken together, these data may support the view that p38 MAPK plays a role in orchestrating cytokine responses and the subsequent cell migration into the bronchial mucosa after LPS exposure in vivo.

In a mouse model of LPS exposure, NF- $\mathrm{kB}$ has been shown to be upregulated in lung tissue homogenates $2 \mathrm{~h}$ after inhalation [20]. In the present study, neither upregulation of total epithelial expression nor nuclear location of NF- $\mathrm{kB}$ or activator protein-1 was observed $3 \mathrm{~h}$ after LPS exposure. Instead, downregulation of nuclear staining of c-Fos, a subunit of the activator protein-1 heterodimer, was noted. p38 MAPK has been described as being related to NF- $\mathrm{\kappa B}$ activation [18]. The association between MAPK and transcription factors in human airway tissue after LPS exposure in vivo has not yet been clarified.

Neutrophil recruitment from the circulation into tissue is dependent on the cell surface expression of vascular endothelial adhesion molecules, such as P-selectin and intercellular adhesion molecule-1 [21]. At this early time point after LPS exposure, adhesion molecules were not upregulated and neutrophil recruitment into the bronchial tissue had not occurred. However, in airway lavage, LPS exposure itself has been shown to induce a two-fold increase in neutrophil numbers [11], indicating that the dose of LPS used was sufficient to induce a significant airway inflammatory response in the distal airway lumen, but not in the proximal bronchial mucosa at this time point. Thus, it is suggested that neutrophil recruitment in the alveoli may follow a different time course compared to the inflammatory response in more proximal bronchial mucosa. The reasons behind the lack of response in the bronchial epithelium may be that bronchial epithelial cells, as shown for other epithelial cells, do not express endogenous CD14 [22], together with TLR-4 one of the major receptors for LPS, or may be due to the different morphological structure in the alveoli compared to the major bronchi. Bronchial epithelial cells may need to be secondarily activated through the release of TNF- $\alpha$ and other cytokines from the LPS-activated alveolar macrophages [23, 24]. In support of this scenario, it has been suggested that LPS stimulates alveolar macrophages primarily via CD14 and TLR-4 [23]. The secretion of TNF- $\alpha$ and other alveolar-macrophage-derived cytokines may consequently be of importance for the LPS-induced inflammatory response in the epithelium, as previously indicated [25]. Furthermore, in the present study, it was shown that LPS challenge induced a pronounced increase in TNF- $\alpha$ levels in both BW and BAL fluid compared to the concentrations below the limit of detection found after placebo inhalation, but no signs of increased TNF- $\alpha$ expression were detected in the proximal bronchial epithelium. Taken together, these data suggest that the LPS-induced airway inflammatory response occurs primarily in the distal airway lumen, with a pronounced inflammatory response as early as $3 \mathrm{~h}$ after LPS inhalation, whereas only early inflammatory signs in terms of increased expression of p38 MAPK were detected in the bronchial epithelium. Further, it is implied that alveolar macrophages may play an inductive role in neutrophil recruitment after LPS exposure.

The present study revealed a small but significant increase in the number of epithelial mast cells. Mast cells secrete multiple cytokines and play an important role in allergic inflammation. The importance of the increase in epithelial mast cell numbers shown after LPS inhalation in the present study is still unclear, but it is conceivable that the response of mast cells to the bacterial component forms part of the innate immune defence against bacterial infections leading to recruitment of inflammatory cells through their ability to secrete cytokines and other inflammatory mediators.

In summary, the present investigation has addressed the early airway mucosal inflammatory responses after exposure to lipopolysaccharides in vivo. It is suggested that the upregulation of phosphorylated p38 mitogen-activated protein kinase and interleukin- 8 in the airway epithelium may represent early regulatory steps in the subsequent development of neutrophilic inflammation in the airway mucosa. However, due to the invasive bronchoscopic procedure used in the present study, it was only possible to address the bronchial epithelial response at one time point after lipopolysaccharide challenge. Complementary research, including investigation at different time points after lipopolysaccharide inhalation, is expected to clarify the kinetics of the local airway mucosal inflammation in response to lipopolysaccharide exposure in humans in vivo.

\section{ACKNOWLEDGEMENTS}

The authors would like to thank A-B. Lundström, H. Bertilsson and L. Skedebrandt for excellent technical assistance.

\section{REFERENCES}

1 Michel O. Systemic and local airways inflammatory response to endotoxin. Toxicology 2000; 152: 25-30. 
2 Michel O, Dentener M, Corazza F, Buurman W, Rylander R. Healthy subjects express differences in clinical responses to inhaled lipopolysaccharide that are related with inflammation and with atopy. J Allergy Clin Immunol 2001; 107: 797-804.

3 Rylander R, Bake B, Fischer JJ, Helander IM. Pulmonary function and symptoms after inhalation of endotoxin. Am Rev Respir Dis 1989; 140: 981-986.

4 Jagielo PJ, Thorne PS, Watt JL, Frees KL, Quinn TJ, Schwartz DA. Grain dust and endotoxin inhalation challenges produce similar inflammatory responses in normal subjects. Chest 1996; 110: 263-270.

5 Sandström T, Bjermer L, Rylander R. Lipopolysaccharide (LPS) inhalation in healthy subjects increases neutrophils, lymphocytes and fibronectin levels in bronchoalveolar lavage fluid. Eur Respir J 1992; 5: 992-996.

6 Michel O, Duchateau J, Plat G, et al. Blood inflammatory response to inhaled endotoxin in normal subjects. Clin Exp Allergy 1995; 25: 73-79.

7 Michel O, Nagy AM, Schroeven M, et al. Dose-response relationship to inhaled endotoxin in normal subjects. Am J Respir Crit Care Med 1997; 156: 1157-1164.

8 Carter AB, Monick MM, Hunninghake GW. Both Erk and p38 kinases are necessary for cytokine gene transcription. Am J Respir Cell Mol Biol 1999; 20: 751-758.

9 Guha M, Mackman N. LPS induction of gene expression in human monocytes. Cell Signal 2001; 13: 85-94.

10 Arbabi S, Rosengart MR, Garcia I, Jelacic S, Maier RV. Priming interleukin 8 production: role of platelet-activating factor and p38. Arch Surg 1999; 134: 1348-1353.

11 Wallin A, Pourazar J, Sandström T. LPS-induced bronchoalveolar neutrophilia; effects of salmeterol treatment. Respir Med 2004; 98: 1087-1092.

12 Blomberg A, Krishna MT, Bocchino V, et al. The inflammatory effects of $2 \mathrm{ppm} \mathrm{NO}_{2}$ on the airways of healthy subjects. Am J Respir Crit Care Med 1997; 156: 418-424.

13 Britten KM, Howarth $\mathrm{PH}$, Roche WR. Immunohistochemistry on resin sections: a comparison of resin embedding techniques for small mucosal biopsies. Biotech Histochem 1993; 68: 271-280.

14 Wilson SJ, Leone BA, Anderson D, Manning A, Holgate ST. Immunohistochemical analysis of the activation of NF- $\kappa B$ and expression of associated cytokines and adhesion molecules in human models of allergic inflammation. I Pathol 1999; 189: 265-272.

15 Montefort S, Gratziou C, Goulding D, et al. Bronchial biopsy evidence for leukocyte infiltration and upregulation of leukocyte-endothelial cell adhesion molecules 6 hours after local allergen challenge of sensitized asthmatic airways. J Clin Invest 1994; 93: 1411-1121.

16 Bosson J, Stenfors N, Bucht A, et al. Ozone-induced bronchial epithelial cytokine expression differs between healthy and asthmatic subjects. Clin Exp Allergy 2003; 33: 777-782.

17 Nick JA, Young SK, Brown KK, et al. Role of p38 mitogenactivated protein kinase in a murine model of pulmonary inflammation. J Immunol 2000; 164: 2151-2159.

18 Hippenstiel S, Soeth S, Kellas B, et al. Rho proteins and the p38-MAPK pathway are important mediators for LPSinduced interleukin-8 expression in human endothelial cells. Blood 2000; 95: 3044-3051.

19 Hashimoto S, Gon Y, Matsumoto K, et al. Selective inhibitor of p38 mitogen-activated protein kinase inhibits lipopolysaccharide-induced interleukin-8 expression in human pulmonary vascular endothelial cells. J Pharmacol Exp Ther 2000; 293: 370-375.

20 Rocksen D, Ekstrand-Hammarstrom B, Johansson L, Bucht A. Vitamin E reduces transendothelial migration of neutrophils and prevents lung injury in endotoxin-induced airway inflammation. Am J Respir Cell Mol Biol 2003; 28: 199-207.

21 Carlos TM, Harlan JM. Leukocyte-endothelial adhesion molecules. Blood 1994; 84: 2068-2101.

22 Backhed F, Meijer L, Normark S, Richter-Dahlfors A. TLR4-dependent recognition of lipopolysaccharide by epithelial cells requires sCD14. Cell Microbiol 2002; 4: 493-501.

23 Philpott DJ, Girardin SE, Sansonetti PJ. Innate immune responses of epithelial cells following infection with bacterial pathogens. Curr Opin Immunol 2001; 13: 410-416.

24 Wong PM, Chung SW, Sultzer BM. Genes, receptors, signals and responses to lipopolysaccharide endotoxin. Scand J Immunol 2000; 51: 123-127.

25 Harmsen AG. Role of alveolar macrophages in lipopolysaccharide-induced neutrophil accumulation. Infect Immun 1988; 56: 1858-1863. 